Website: http://revistas.lamolina.edu.pe/index.php/acu/index

(C) Universidad Nacional Agraria La Molina, Lima - Perú

\title{
Productividad del capital humano para incrementar el ingreso per cápita de la población económicamente activa (PEA) a nivel nacional y regional: caso peruano período $2008-2015$
}

\author{
Productivity of the human capital to increase the income of the economically active population \\ (PEA) at the national and regional level: peruvian case period $2008-2015$
}

\author{
Claudio Stefano S. Castagnino Pastor ${ }^{1} \&$ Agapito Linares Salas ${ }^{2}$
}

\begin{abstract}
Resumen
El objetivo de este estudio fue deterrminar el grado de incidencia de la productividad del capital humano tomando en cuenta los cambios en el PBI respecto a las inversiones realizadas por el gobierno en el recurso humano que interviene en la producción, de igual manera la incidencia sobre la Población Económicamente Activa total (PEA) con nivel de educación superior y la convergencia de los ingresos per cápita entre las regiones de nuestro país. Se estudia las veinticuatro regiones del Perú para los años del 2008 al 2015. La metodología de la investigación fue cuantitativa y emplea modelos econométricos, basándose en el modelo de Solow Extendido y su Convergencia. Los resultados demuestran que la productividad del capital humano $\left(s_{h}\right)$ es el factor principal que incide en el incremento del ingreso per cápita y reduce la brecha de los ingresos (convergencia) entre las diferentes regiones del Perú. Por lo tanto, consideramos que las políticas del gobierno central y la de los gobiernos regionales deben orientarse a mejorar el capital humano, factor determinante para el incremento del ingreso per cápita y acelera la convergencia de los ingresos per cápita.
\end{abstract}

Palabras clave: Productividad del Capital Humano; Convergencia; Modelo de Solow; Solow Perú; Stata.

\begin{abstract}
The objective of this study was to determine the degree of incidence of the productivity of human capital, taking into account the changes in the GDP with respect to the investments made by the government in the human resources that intervene in the production, as well as the incidence on the Total Economically Active Population (EAP) with a higher education level and the convergence of per capita income between the regions of our country. The twenty-four regions of Peru are studied for the years of 2008 to 2015. The research methodology is econometric, based on the extended Solow model and Convergence. The results show that human capital $\left(\mathrm{s}_{h}\right)$ is the main factor increases the income and reduces the income gap (convergence) between the different regions of Peru. Therefore, the policies of the central and regional government should be oriented towards improving human capital, a determining factor for the increase of the income and accelerating convergence.
\end{abstract}

Keywords: Productivity of Human Capital; Convergence; Solow model; Solow Peru; Stata.

\section{Introdución}

La incidencia de la productividad del capital humano sobre el crecimiento económico de las sociedades es un aspecto importante para el bienestar de sus miembros. De hecho, para Smith la Economía Política gozaría de "buen funcionamiento" siempre y cuando la ética (como prudencia y cuidado personal), la economía (el logro del bienestar económico para cada ciudadano) y la política (protección de los derechos y justicia conmutativa por parte de las leyes y los guardianes del sistema) estuvieron interrelacionadas $\mathrm{y}$ fueron inseparables $\mathrm{y}$ necesarias (Pique, 2018).

En la segunda mitad del siglo pasado un fenómeno aparentemente inexplicable apareció, fue el crecimiento impresionante de las economías emergentes, como: China (9,65\% en promedio desde 1980 al 2015), Corea del Sur (9\% en promedio desde 1961 al 2000), y Perú $(5,17 \%$ en promedio desde el 2000 al 2015), entre otros (The World Bank, 2017). No solo fue impresionante por las cifras altas de crecimiento sino porque fueron países con limitada productividad en su capital físico, escaso capital humano calificado, casi nulo desarrollo tecnológico e instituciones con bajo desarrollo.

La respuesta se encuentra con la ayuda del modelo de Solow (1956), premio Nobel, presentado en su obra: "Una Contribución a la Teoría del Crecimiento Económico", posteriormente, lo desarrolla empíricamente, en la obra: "Una Contribución Empírica del Crecimiento Económico", donde demuestra porque algunos países se hacen ricos y otros países se hacen pobres.

Por la limitada información disponible al convertirse en una herramienta importante en la toma de decisiones más eficientes en relación a la productividad del capital humano y los cambios en el PBI, la inversión en capital

\footnotetext{
conomista; Oracle Certified Professional, Java Programmer y CompTIANetwork+Certified.

${ }^{2}$ Profesor Principal, Facultad de Economía y Planificación, Universidad Nacional Agraria la Molina, Lima, Perú. Email: alinares@lamolina.edu. pe
} 
físico y crecimiento de la población, gestión de los gobiernos regionales y gobierno central. Los resultados obtenidos permitirán el mejoramiento del bienestar de los peruanos y disminuir las brechas de ingreso per cápita de la PEA entre las diferentes regiones. El sector privado se verá beneficiado al incrementarse y desarrollarse los mercados de consumo.

El objetivo del presente estudio fue determinar el grado de incidencia de la PEA calificada con educación universitaria y mayor productividad en el incremento del PBI per cápita en el Perú y a nivel regional el grado de convergencia entre las regiones explicadas por la productividad del capital humano.

\section{Materiales y métodos}

Los materiales y métodos utilizados se materializan en la formulación del modelo de Solow Extendido y el de Convergencia, para lo cual se empleó la información disponible de los años 2008 al 2015 de las regiones del Perú excepto la provincia constitucional del Callao. Se utilizó la econometría como metodología; aplicando MCO (Mínimo Cuadro Ordinario) y la prueba de Breusch-Pagan.

Mediante el Modelo de Solow Extendido y el de Convergencia se determina: la constante (A) que representa el avance tecnológico, la variable $\left(s_{k}\right)$ el capital físico, la variable $\left(s_{h}\right)$ capital humano, variables que afectan positivamente al ingreso per cápita; sin embargo, el aumento de la población (n) en este modelo afecta negativamente el crecimiento del ingreso per cápita en el período de estudio. Según las regresiones formuladas y los casos planteados explican el ingreso per cápita en un 61 y $67 \%$; en cambio mediante la regresión de convergencia se explica la reducción de las brechas de los ingresos per cápita entre regiones en un $42 \%$.

Como resultado se observa que efectivamente la productividad del capital humano es la principal variable explicativa, seguida del capital físico para el mejoramiento del ingreso per cápita de los peruanos y la convergencia de los ingresos per cápita entre las regiones durante el período del 2008 al 2015.

El establecimiento de las relaciones causa-efecto entre las variables endógenas y exógenas, el crecimiento per cápita de los ingresos, la productividad de los factores, la formación del capital físico y los niveles tecnológicos, proporcionan el marco teórico del crecimiento económico de los países y al interior de las regiones.

\section{Modelo de Solow y Solow Extendido}

De acuerdo al modelo de Solow, la economía puede alcanzar dos estados estacionarios: la producción como el capital per cápita son iguales en el estado estacionario, el segundo estado estacionario se alcanza por la forma que adopte la función de producción neoclásica (relación producción y recursos); el supuesto decisivo para que ello suceda es el relacionado con la productividad marginal del capital, que tiende a cero cuando el capital físico tiende a infinito, pues de no existir este supuesto no se alcanzaría dicho estado estacionario (Plata et al., 2017).

El modelo de Solow revela que las naciones son más ricas porque invierten más y tienen menores tasas de crecimiento de la población, estos factores son: la tasa de ahorro (s), la tasa de crecimiento de la población (n) la inversión física (IBF), la acumulación del capital (K*), el nivel de producción (PBI), y la inversión de compensación al desgaste del capital $(\delta)$ (Linares, 2012).

Markiew et al. (1992) desarrollo el modelo de Solow Extendido adicionando el capital humano, los resultados demostraron la importancia del capital humano por su productividad en el crecimiento de los países. El modelo de Solow basado en el modelo de producción de CobbDouglas (CD), tiene los siguientes supuestos de acuerdo a nuestro objetivo: Economía de Mercado donde se produce un bien y donde solo se consume e invierte. La relación de Capital/Producto es endógena y flexible. La fuerza de trabajo crece a una tasa constante y exógena. Existe un mercado de competencia perfecta. La tasa de ahorro, la tasa de crecimiento de la población y progreso tecnológico son exógenos.

Regresión de Solow Extendido:

$Y(t)=K(t) \alpha \mathrm{H}(t) \beta(\mathrm{A}(t) \mathrm{L}(\mathrm{t}))^{1-\alpha-\beta}$

Para todo $0<\alpha<1, t$ es la unidad de tiempo.

Donde:

Y: Producción.

K: Capital Físico.

H: Porcentaje del capital humano. A: Nivel de Tecnología.

L: Fuerza Laboral o total usado $\alpha$ : Elasticidad de K.

$\beta$ : Elasticidad de $\mathrm{H}$.

$\alpha-\beta$ : Elasticidad $n$.

La acumulación de capital físico y del capital humano se determinan por las siguientes ecuaciones:

$\mathrm{k}(\mathrm{t})=\mathrm{s}_{\mathrm{k}} \mathrm{y}(\mathrm{t})-(\mathrm{n}+\mathrm{g}+\delta) \kappa(\mathrm{t})$

$h(t)=s_{h} y(t)-(n+g+\delta) h(t)$

Para todo:

$\mathrm{s}_{\mathrm{k}}$ : Es la fracción del ingreso per cápita invertido en capital físico.

$\mathrm{S}_{\mathrm{h}}$ Es la fracción del ingreso per cápita invertido en capital humano.

En forma logarítmica.

$\ln (\mathrm{Y}(\mathrm{t}) / \mathrm{L}(\mathrm{t}))=\ln \mathrm{A}(0)+\mathrm{gt}-((\alpha+\beta) /(1-\alpha-\beta)) \ln (\mathrm{n}+\mathrm{g}+\delta)+\alpha /(1-$ $\alpha-\beta) \ln \left(\mathrm{s}_{\mathrm{k}}\right)+\beta /(1-\alpha-\beta) \ln \left(\mathrm{s}_{\mathrm{h}}\right)$

De esta forma el modelo de Solow predice que: "El crecimiento de las economías será finito y en consecuencia las economías convergen". 


\section{Convergencia}

El modelo de Solow predice la convergencia solo después de controlar los determinantes del estado estacionario, un fenómeno que podría llamarse "condicional de convergencia (Jiang, 2013).

Expresado en la siguiente ecuación logarítmica de convergencia:

$\mathrm{d} \ln (y(t) / \mathrm{dt})=\lambda\left[\ln \left(y^{*}\right)-\ln (y(t))\right]$

Donde endógenamente la tasa de convergencia se determina mediante la siguiente relación:

$\lambda=(\mathrm{n}+\mathrm{g}+\delta)(1-\alpha-\beta)$

$\lambda$, es la tasa de convergencia, si por citar la tasa de convergencia sería igual a 0,02 , significaría que la economía estaría a treinta cinco años del estado estacionario, y si fuera de 0,04 estaría alrededor de unos diecisiete años, lo que significa que a medida que aumenta el coeficiente de convergencia, menor es el número de años para alcanzar la igualdad del ingreso per cápita entre las regiones. $y^{*}$, es el estado estacionario de la renta por efectivo trabajador dado en la ecuación 4 (Markiew et al., 1992).

El modelo sugiere una regresión para el estudio de la tasa de convergencia.

$$
\ln (y(t))=\left(1-\mathrm{e}^{-\lambda t}\right) \ln \left(y^{*}\right)+\mathrm{e}^{-\lambda t} \ln (\mathrm{y}(0))
$$

Donde:

$y(0)$ : Es el ingreso por trabajador en el período inicial 0.

$\ln (y(t))-\ln (y(0))=\left(1-\mathrm{e}^{-\lambda t}\right) \ln \left(y^{*}\right)-\left(1-\mathrm{e}^{-\lambda t}\right) \ln (y(0))$

Sustituyendo por:

$\ln (y(t))-\ln (y(0))=\left[\left(\left(1-\mathrm{e}^{-\lambda t}\right) \alpha /(1-\alpha-\beta)\right) \ln \left(\mathrm{s}_{\mathrm{k}}\right)\right]+\left[\left(1-\mathrm{e}^{-\lambda t}\right) \beta /(1-\right.$ $\left.\alpha-\beta) \ln \left(s_{h}\right)\right]-\left[\left(\left(1-\mathrm{e}^{-\lambda t}\right)(\alpha+\beta) /(1-\alpha-\beta)\right)\right] \ln (\mathrm{n}+\mathrm{g}+\delta)-\left(1-\mathrm{e}^{-\lambda t}\right) \ln ($ $\mathrm{y}(0))$

La convergencia es más fácil de estimar cuando los países, estados, regiones o departamentos tienen en común un mismo o parecido sistema institucional.

\section{Metodología de investigación}

La metodología de investigación para el presente trabajo fue el método econométrico. Se obtuvo la información de las variables desde fuentes secundarias. Del marco teórico se extrajo las regresiones cuatro (4) y nueve (9).

Donde el Ingreso per cápita (y) es igual al PBI per cápita de la PEA. La diferencia de ingreso per cápita entre 2008 y 2015 es igual a la diferencia del PBI per cápita de la PEA para el mismo período. Población (n) es igual a la tasa de crecimiento de la población promedio. El capital humano $\left(s_{h}\right)$ es igual al promedio porcentual de la PEA con educación superior universitario. La inversión en capital físico $\left(s_{k}\right)$ es igual al gasto público promedio por estudiante del nivel superior universitario.

Este modelo se enfoca en el capital humano en la forma de inversión en la educación versus la inversión en la población sin educación. Se usa el proxy de la tasa de capital humano, el porcentaje de la PEA con educación universitaria en las regiones del Perú, asumiendo que ( $\mathrm{g}$ $+\delta$ ) es igual a 0,05 a falta de información local y por ser un valor que no influye sustancialmente en el resultado (Markiew et al., 1992).

\section{Variables principales para la investigación}

Se procesó información de las regiones del Perú, para los años 2008 al 2015, en el cual se desarrolló y definió según INEI, las siguientes variable: El PBI per cápita de la PEA Regional del año 2008. El PBI per cápita de la PEA Regional del año 2015. La diferencia entre PBI per cápita de la PEA Regional de los años 2008 y 2015. Tasa de Crecimiento de la Población Promedio (n). EL Promedio Porcentual de la PEA Regional con Educación Superior Universitaria $\left(s_{h}\right)$. El Gasto Publico Regional Promedio por Alumno Superior Universitario $\left(s_{k}\right)$.

Se evaluó el ingreso per cápita (y2008) es igual al PBI per cápita de la PEA del año 2008. El ingreso per cápita $\left(y_{2015}\right)$ es igual al PBI per cápita de la PEA del año 2015. La diferencia de ingreso per cápita $\left(y_{2015}-y_{2008}\right)$ es igual al ingreso per cápita $\left(y_{2015}\right)$ menos el ingreso per cápita $\left(y_{2008}\right)$.

\section{Base de Datos: una breve explicación}

La información para la aplicación de este modelo se ha obtenido del Instituto Nacional de Estadística e Informática (INEI), del Sistema Integrado de Administración Financiera del Sector Público (SIAF-SP), del Ministerio de Economía y Finanzas, y el Banco Mundial (World Bank). El PBI per cápita de la PEA de los años 2008 y 2015 se obtuvo del PBI a precios constantes del 2007 entre la PEA nacional y la tasa de crecimiento de la población promedio, tomando como promedio en este caso los años 2008 al 2015. Se obtuvo también la tasa de crecimiento anual de la población, el promedio porcentual de la PEA con educación universitario. De igual forma se estimó el promedio de los años 2008 al 2015 el porcentaje de población en edad de trabajar con educación universitaria y el gasto público promedio por alumno universitario. Fue necesario determinar el promedio durante el periodo 2008 al 2015 de gasto público en educación por alumno universitario a precios corrientes del 2007, no se consideró la información de Lima Metropolitana sino Lima provincias, todas las regiones del Perú sin la provincia constitucional del Callao por falta de información, los resultados no fueron redondeados y la información monetaria real expresada en soles peruanos del 2007. 
Tabla 1. Variables

\begin{tabular}{|c|c|c|}
\hline $\begin{array}{l}\text { Nombre de } \\
\text { la Variable } \\
\text { en Teoría }\end{array}$ & $\begin{array}{c}\text { Nombre de la Variable } \\
\text { en Stata (forma no } \\
\text { logarítmica) }\end{array}$ & $\begin{array}{c}\text { Nombre de la Variable en } \\
\text { Archivo (Anexo 1) }\end{array}$ \\
\hline \multicolumn{3}{|c|}{ Variables Endógenas } \\
\hline \multirow{2}{*}{\multicolumn{2}{|c|}{$\begin{array}{c}y_{2008} \text { pbi_pea_percapita_2008 } \\
y_{2015} \text { pbi_pea_percapita_2015 } \\
\text { Dif_2015_18 }\end{array}$}} & PBI_PEA \\
\hline & & PBI \\
\hline \multicolumn{3}{|c|}{$y_{2015} y_{2008}$ Variables Exógenas } \\
\hline & tasa_crec_prom & \%Tasa_Crec_Prom_Pob \\
\hline & _univ & $\begin{array}{l}\text { Promedio \%_PEA_EDU_ } \\
\text { UNIV }\end{array}$ \\
\hline & $\begin{array}{l}\text { gasto_pub_prom_per_ } \\
\text { alumn_univ }\end{array}$ & $\begin{array}{l}\text { Gasto_Pub_Prom_per_ } \\
\text { Alumn_Univ }\end{array}$ \\
\hline
\end{tabular}

La variable $\left(y_{2008}\right)$, es la variable exógena para la regresión de la Convergencia. Debe añadirse que toda variable en su forma logarítmica se antepone (ln) más su nombre de la variable en para los programas realizados en Stata en su forma no logarítmica (Castagnino, 2018).

\section{Equipos Usados y Procedimientos}

Se utilizó el software estadístico Stata versión 13 de prueba, aplicando los programas de Castagnino (2018) anexos 1, 2 y 3 ; la econometría para el desarrollo de la teoría.

\section{Resultados y discusión}

\section{Regresiones}

Las regresiones simples se ejecutaron con el método de Mínimo Cuadro Ordinario (MCO) o Least Square $(L S)$.

\section{Caso I}

Tabla 2. Regresión del Caso I de $y_{2008}=\mathrm{c}_{0}-\mathrm{c}_{1} \mathrm{n}+\mathrm{c}_{2} \mathrm{~s}_{\mathrm{h}}+\mathrm{c}_{3} \mathrm{~s}_{\mathrm{k}}$

\begin{tabular}{|c|c|c|c|c|c|c|c|c|}
\hline Source & SS & df & MS & \multirow{2}{*}{\multicolumn{3}{|c|}{$\begin{array}{l}\text { Number of obs }= \\
\begin{array}{ll}\mathbb{F}(3, \quad 20) & =13 . \\
\text { Prob }>F & =0.00\end{array}\end{array}$}} & \\
\hline Model & 5.86934408 & \multicolumn{2}{|c|}{31.95644803} & & & & & \\
\hline Residual & 2.99238529 & 20 & .149619264 & \multicolumn{5}{|c|}{ R-squared $=0.6623$} \\
\hline & & & & \multicolumn{5}{|c|}{ Adj R-squared $=0.6117$} \\
\hline Total & 8.86172937 & 23 & .385292581 & & MSE & $=33 x+3$ & & \\
\hline \multicolumn{3}{|c|}{ In_pbi_pea_percapita_2008 } & Coef. & Std. Err. & $\mathrm{t}$ & $\mathrm{P}\rangle|\mathrm{t}|$ & [95\% Conf. & Interval] \\
\hline \multirow{2}{*}{\multicolumn{3}{|c|}{$\begin{array}{l}\text { In_tasa_crec_prom pob } \\
\text { In_promedio pea_edu univ }\end{array}$}} & -.0824942 & 1.183474 & -0.07 & 0.945 & -2.551178 & 2.38619 \\
\hline & & & 1.197429 & .2412292 & 4.96 & 0.000 & .6942343 & 1.700625 \\
\hline \multicolumn{3}{|c|}{ In gasto pub prom per alumn univ } & .7371727 & .2328884 & 3.17 & 0.005 & .2513761 & 1.222969 \\
\hline & & ions & 5.649689 & 4.174216 & 1.35 & 0.191 & -3.057574 & 14.35695 \\
\hline
\end{tabular}

Representación de la regresión del Caso I.

$y_{2008}=5,650-0,083 \mathrm{n}+1,197 s_{h}+0,737 s_{k}$

Interpretación de los coeficientes estimados:

Cuando la tasa de crecimiento poblacional aumenta en $1 \%$ en promedio el ingreso per cápita disminuye en $0,083 \%$. Cuando la productividad del capital humano aumenta en $1 \%$ en promedio el ingreso per cápita aumenta en 1,197 $\%$. Cuando el capital físico aumenta en $1 \%$ en promedio el ingreso per cápita aumenta en $0,737 \%$.

\section{Caso II}

Tabla 3. Regresión del caso II de $y_{2015}=\mathrm{c}_{0}-\mathrm{c}_{1} \mathrm{n}+\mathrm{c}_{2} \mathrm{~s}_{h}+\mathrm{c}_{3} \mathrm{~s}_{\mathrm{k}}$

\begin{tabular}{|c|c|c|c|c|c|c|c|c|c|}
\hline Source & \multicolumn{3}{|c|}{ SS } & MS & & \multirow{2}{*}{\multicolumn{4}{|c|}{$\begin{array}{l}\text { Number of obs }= \\
F(3, \quad 20)=16.69\end{array}$}} \\
\hline & \multirow{2}{*}{\multicolumn{2}{|c|}{4.66727145}} & & 1.55575715 & & & & & \\
\hline Model & & & 3 & $1.555 / 5 / 15$ & & \multicolumn{4}{|c|}{ Prob > F $\quad=0.0000$} \\
\hline Residual & \multicolumn{2}{|c|}{1.86464191} & 20 & .093232095 & & \multicolumn{4}{|c|}{ R-squared $\quad=0.7145$} \\
\hline & \multirow{2}{*}{\multicolumn{2}{|c|}{6.53191335}} & & & & \multicolumn{4}{|c|}{ Adj R-squared $=0.6717$} \\
\hline Total & & & 23 & .283996233 & & \multicolumn{2}{|c|}{ Root MSE } & \multicolumn{2}{|c|}{$=.30534$} \\
\hline \multicolumn{2}{|c|}{ In_pbi_pea_pe 2015} & \multicolumn{2}{|c|}{ Coef. } & Std. Err. & $\mathrm{t}$ & $P>|t|$ & [95\% & Conf. & Interval] \\
\hline \multirow{3}{*}{\multicolumn{2}{|c|}{$\begin{array}{l}\text { In_tasa_crec_pro b } \\
\text { ln_promedio_pea_ } \sim v \\
\text { ln_gasto_pub_pro v }\end{array}$}} & \multicolumn{2}{|c|}{-.160143} & .934217 & -0.17 & 0.866 & -2.10 & 8886 & 1.7886 \\
\hline & & \multicolumn{2}{|c|}{1.123968} & .1904227 & 5.90 & 0.000 & & 7529 & 1.521183 \\
\hline & & \multicolumn{2}{|c|}{.5779217} & 1838386 & 3.14 & 0.005 & & 4411 & 9614024 \\
\hline \multicolumn{2}{|c|}{ cons } & \multicolumn{2}{|c|}{6.850726} & 3.295065 & 2.08 & 0.051 & -.022 & 6583 & 13.72411 \\
\hline
\end{tabular}

Representación de la Regresión del caso II.

$y_{2015}=6,851-0,160 \mathrm{n}+1,224 \mathrm{~s}_{\mathrm{h}}+0,578 \mathrm{~s}_{\mathrm{k}}$

Interpretación de los coeficientes estimados:

Cuando la tasa de crecimiento poblacional aumenta en $1 \%$ en promedio el ingreso per cápita disminuye en $0,160 \%$. Cuando la productividad del capital humano aumenta en $1 \%$ en promedio el ingreso per cápita aumenta en $1,224 \%$. Cuando el capital físico aumenta en $1 \%$ en promedio el ingreso per cápita aumenta en $0,578 \%$.

De las Tabla 2 y Tabla 3 se observa que los ajustado (Adj R-squared) son 0,6117; 0,6717; lo que representa un fuerte apoyo al modelo de Solow Extendido. El coeficiente (cons) en Caso I no es significativo al tener una probabilidad $(\mathrm{P}>|\mathrm{t}|=0,191)$, mayor que 0,05 , en el caso II es significativo $(\mathrm{P}>|\mathrm{t}|=0,051)$. Las probabilidades de los In_tasa_crec_prom_pob $(\mathrm{P}>|\mathrm{t}|=0,945 ; \mathrm{P}>|\mathrm{t}|=0,866)$ en los dos casos no son significativas; ln_promedio_pea edu_univ $(\mathrm{P}>|\mathrm{t}|=0,000)$ y en cambio hay significancia en: ln_gasto_pub_prom_per_alumn_univ $(\mathrm{P}>|\mathrm{t}|=0,005)$.

\section{Caso: Modelo de Convergencia}

Tabla 4. Regresión de $y_{2015}-y_{2008}=\mathrm{c}_{0}+\mathrm{c}_{1} y_{2008}+\mathrm{c}_{2} \mathrm{n}+\mathrm{c}_{3} \mathrm{~s}_{k}+$

\begin{tabular}{|c|c|c|c|c|c|c|c|c|}
\hline \multicolumn{9}{|c|}{$\mathrm{c}_{4} \mathrm{~s}_{h}$} \\
\hline Source & SS & df & MS & \multirow{2}{*}{\multicolumn{5}{|c|}{ 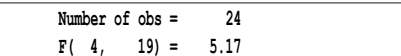 }} \\
\hline & & & & & & & & \\
\hline Model & .330152869 & 4 & .082538217 & \multicolumn{5}{|c|}{ Prob $>F \quad=0.0054$} \\
\hline Residual & .303337509 & 19 & .015965132 & \multirow{3}{*}{\multicolumn{3}{|c|}{$\begin{array}{l}\text { R-squared = } \\
\text { Adj R-squared = }\end{array}$}} & & \\
\hline & & & & & & & & \\
\hline Total & .633490379 & 23 & .02754306 & & & & & \\
\hline \multicolumn{3}{|c|}{ In__if_2015_18 } & Coef. & Std. Err. & $\mathrm{t}$ & $P>|t|$ & [95\% Conf & Interval] \\
\hline \multicolumn{3}{|c|}{ In_pbi_pea_percapita_2008 } & -.277671 & .0730428 & -3.80 & 0.001 & -.4305514 & -.1247907 \\
\hline \multicolumn{3}{|c|}{ In_tasa_crec_prom_pob } & -.100555 & .3866376 & -0.26 & 0.798 & -.9097968 & .7086867 \\
\hline \multicolumn{3}{|c|}{ In promedio pea edu univ } & .2590297 & .1177252 & 2.20 & 0.040 & .0126281 & .5054313 \\
\hline \multicolumn{3}{|c|}{ In gasto pub prom per_alumn univ } & .0454405 & .0932023 & 0.49 & 0.631 & -.1496341 & 2405151 \\
\hline \multicolumn{3}{|c|}{ cons } & 2.769792 & 1.424617 & 1.94 & 0.067 & -.2119656 & 5.75155 \\
\hline
\end{tabular}

El coeficiente del ingreso per cápita del 2008 (- 0,28), con un ajustado del 0,42 , nos indica una fuerte evidencia de convergencia de los ingresos per cápita hacia el 2015.

\section{Propósito y significancia}

De la Tabla 2 y Tabla 3 de la variable ln_promedio_pea 
edu_univ que representa la productividad del $\mathrm{s}_{\mathrm{h}}$ capital humano, en el presente trabajo se obtuvo los siguientes resultados que forma resumida se pueden visualizar en la siguiente Tabla 5.

Tabla 5. Resumen del capital humano

\begin{tabular}{ccc}
\hline Casos & Coeficiente & Probabilidad $(\mathrm{p})$ \\
\hline Caso I & 1,197 & $<0,05$ \\
Caso II & 1,224 & $<0,05$ \\
\hline
\end{tabular}

Significando que la productividad del capital humano es la variable principal que contribuye realmente al incremento del PBI per cápita de la PEA o ingreso per cápita (y), al poseer el mayor coeficiente positivo con una $\mathrm{p}<0,05$ para ambos casos. De la tabla relativa al análisis de la Convergencia se obtiene:

Tabla 6. Resumen de convergencia

\begin{tabular}{lcc}
\hline $\begin{array}{l}\text { Variables Exógenas } \\
\text { adicionales }\end{array}$ & Coeficiente de $y_{2008}$ & $R^{2}$ Ajustado \\
\hline $\mathrm{n}, \mathrm{s}_{\mathrm{k}}, \mathrm{sh}$ & $-0,277$ & 0,42 \\
\hline
\end{tabular}

Los valores de la convergencia se acrecientan cuando los coeficientes de las variables son más negativos y el R2 se aproxima a 1. Se puede afirmar por lo tanto que en Perú existe convergencia entre las regiones y se asevera que en presencia del capital humano $\left(s_{h}\right)$, la convergencia es más rápida $(-0,277)$, en otros términos, la brecha de PBI per cápita entre las regiones del Perú disminuirá con mayor rapidez si la productividad del capital humano se incrementa.

De la investigación de Markiew et al. (1992) se considera como capital humano a la población cursando estudios de educación secundaria entre las edades de 12 a 17, es una variable imperfecta porque no todo el capital humano es parte de la PEA. En esta investigación el capital humano y la población con estudios de educación universitaria constituyen parte de la PEA para el caso de esta investigación.

\section{Conclusiones}

La PEA con Educación Universitaria $\left(s_{h}\right)$ es la de mayor productividad, variable que más acrecienta el PBI per cápita, seguida por la productividad de la población con estudios universitarios $\left(s_{k}\right)$. Los resultados de la regresión (12) y la regresión (13), así lo demuestran, es decir que cuando la productividad del capital humano aumenta en $1 \%$ en promedio el ingreso per cápita aumenta en $1,224 \%$ y el ingreso per cápita aumenta en $0,578 \%$.

Existe convergencia del PBI per cápita de la PEA entre las regiones del Perú y, la PEA con Educación Universitaria, variables con mayor incidencia en la disminución de la brecha entre las regiones de mayores ingresos per cápita relativos y las regiones de más bajos ingreso per cápita para el período 2008 - 2015. Es el caso, por ejemplo: Apurímac con menor PBI per cápita en 2008 creció cuatro veces más que Moquegua con un mayor PBI per cápita en el 2015. 282
Utilizando el modelo de Solow Extendido se demuestra que la productividad del capital humano $\left(s_{h}\right)$ incrementa el ingreso per cápita de los peruanos y también disminuye las diferencias de ingreso per cápita entre residentes de las diferentes regiones del Perú, lo que significa que mantener altas las tasas de productividad es esencial para contar con un uso sostenible de los recursos productivos y para contribuir con el crecimiento regional y nacional.

\section{Recomendaciones de políticas}

El gobierno central y los gobiernos regionales deben impulsar el crecimiento económico desde de las regiones de menores ingresos per cápita, por ser las de mayor crecimiento económico promedio para el período, con una mayor inversión en capital físico para incrementar la productividad del capital humano con educación universitaria, proceso que se viene denominando como "crecimiento económico desde adentro".

\section{Literatura citada}

Castagnino, S.2018. Mejoramiento de Capital Humano Para el Incremento Del Ingreso Per Cápita De la PEA Caso Peruano Años 2008 y 2015.49, 56,71,73,76, 77pp. Universidad Nacional Agraria La Molina, Lima, Perú.

ESCALE [Unidad de Estadística Educativa - Ministerio de Educación]. 2016. Gasto público en educación por alumno, superior universitaria -soles corrientes (en línea). Disponible en: http://escale.minedu.gob.pe/.

INEI [Instituto Nacional de Estadística e Informática]. 2016. Dirección Nacional de Cuentas Nacionales. Indicadores: PBI a precios constantes 2007, PEA, PBI a precios corrientes, Tasa de crecimiento promedio anual de la población. Disponible en: https://www.inei. gob.pe.

Jiang, Y. 2013. Openness, Economic Growth and Regional Disparities: The Case of China. 32pp. Springer, Berlin, Heidelberg.

Linares, A. 2012. Teoría y Política Macroeconómica en una Economía Abierta. 326pp. Universidad Nacional Agraria La Molina, Lima, Perú.

Mankiw, G.; Romer, D.; Weil, D. 1992. A Contribution to the Empirics of Economic Growth. 408, 413, 415pp. The Quarterly Journal of Economics, 107:407-437

Piqué, P. 2018. La Enseñanza del Proyecto Filosófico de Adam Smith En La Historiografía del Pensamiento. Pp 99. Praxis Filosófica Nueva serie, No. 46 enero-junio 2018: 89110 DOI: 10.25100/pfilosófica.v0i46.6150\}

Plata, L.; González, P.; Calderón, E. 2017. La función de crecimiento de Richard y los modelos de crecimiento neoclásicos. EconoQuantum [online] 14 (1): 99-118.

Solow, R. 1956. A Contribution to the Theory of conomic Growth", The Quarterly Journal of Economics 70. The World Bank. 2017. Índices: PBI per cápita de China, Corea de Sur, Filipinas y Perú (en línea). Disponible en: https://data.worldbank.org/indicator/NY.GDP. PCAP.CD. 
Anexos

Anexo 1:Informacion para el desarrollo de las regresiones de esta investigación (data)

\begin{tabular}{lrrrrr}
\hline \multicolumn{1}{c}{ AÑO } & \multicolumn{1}{c}{$\begin{array}{c}\text { PBI_PEA } \\
\text { percapita_2008 }\end{array}$} & $\begin{array}{c}\text { PBI_PEA } \\
\text { percapita_2015 }\end{array}$ & $\begin{array}{c}\text { \%Tasa_Crec_Prom } \\
\text { _Pob }\end{array}$ & $\begin{array}{c}\text { Promedio\% PEA_ } \\
\text { EDU_UNIV }\end{array}$ & $\begin{array}{c}\text { Gasto_Pub_Prom_per_ } \\
\text { Alumn_Univ }\end{array}$ \\
\hline Amazonas & 8824,64 & 12182,87 & 0,513 & 6,900 & 9324,484076 \\
Ancash & 28431,69 & 28074,18 & 0,588 & 13,213 & 6015,728353 \\
Apurímac & 7079,67 & 9924,48 & 0,563 & 10,363 & 6005,238493 \\
Arequipa & 30527,65 & 34090,84 & 1,075 & 22,150 & 3346,583881 \\
Ayacucho & 10317,85 & 14637,12 & 1,175 & 10,263 & 3734,702918 \\
Cajamarca & 11061,25 & 13150,43 & 0,450 & 7,613 & 4406,494506 \\
Cusco & 16394,25 & 27424,19 & 0,675 & 13,275 & 5020,545999 \\
Huancavelica & 11257,67 & 12672,34 & 0,825 & 6,038 & 4320,486455 \\
Huánuco & 8177,28 & 10934,07 & 0,850 & 11,888 & 4530,330614 \\
Ica & 27634,72 & 38035,00 & 1,075 & 17,838 & 5302,720882 \\
Junín & 14957,87 & 20100,44 & 0,738 & 15,138 & 4450,450044 \\
La Libertad & 18696,66 & 21220,42 & 1,288 & 14,138 & 4210,540807 \\
Lambayeque & 12308,77 & 16966,53 & 0,888 & 11,863 & 3837,910171 \\
Lima & 31631,21 & 41338,25 & 1,500 & 22,000 & 6388,291951 \\
Loreto & 16019,75 & 16707,60 & 1,213 & 9,563 & 6449,618177 \\
Madre de Dios & 28763,34 & 29532,52 & 2,638 & 14,075 & 8031,646191 \\
Moquegua & 93063,13 & 84088,02 & 1,075 & 17,513 & 16802,860720 \\
Pasco & 40564,74 & 32499,96 & 0,800 & 15,400 & 6237,287674 \\
Piura & 15793,06 & 20608,47 & 0,825 & 9,550 & 5380,192001 \\
Puno & 8561,58 & 10665,36 & 0,900 & 13,463 & 4590,141878 \\
San Martin & 8960,55 & 12897,96 & 1,488 & 8,175 & 5600,275310 \\
Tacna & 29146,09 & 36701,10 & 1,388 & 11,938 & 5793,617898 \\
Tumbes & 15766,14 & 19723,22 & 1,513 & 10,000 & 9942,807899 \\
Ucayali & 13407,63 & 15290,39 & 1,375 & 6303,537305 \\
\hline & & & &
\end{tabular}

Fuente: INEI (2017) y ESCALE (2016).

. Producto Bruto Interno a precios constantes de 2007(Miles de soles de 2007).

Población económicamente activa (Personas).

*Gasto público en educación por alumno, superior universitario (soles corrientes).

Tasa de crecimiento promedio anual de la población (Porcentaje).

Porcentaje de la población económicamente activa con educación superior universitaria (Porcentaje).

Producto Bruto Interno a precios corrientes (Miles de soles).

Producto Bruto Interno per cápita a precios constantes de 2007 (Soles).

Corrido a precios soles del 2007.

Anexo 2. Capital Físico

\begin{tabular}{|c|c|c|}
\hline $\begin{array}{l}\text { Crecimiento } \\
\text { Porcentual }\end{array}$ & $\begin{array}{c}\text { Gasto Publico Alumno } \\
\text { Universitario Nacional (soles } \\
\text { 2007) }\end{array}$ & $\begin{array}{l}\text { PBI per cápita } \\
\text { (soles 2007) }\end{array}$ \\
\hline 2008 & $15,94 \%$ & $7,90 \%$ \\
\hline 2009 & $-0,02 \%$ & $-0,03 \%$ \\
\hline 2010 & $26,47 \%$ & $7,12 \%$ \\
\hline 2011 & $0,54 \%$ & $5,13 \%$ \\
\hline 2012 & $8,30 \%$ & $4,94 \%$ \\
\hline 2013 & $11,98 \%$ & $4,68 \%$ \\
\hline 2014 & $13,96 \%$ & $1,23 \%$ \\
\hline 2015 & $19,82 \%$ & $2,14 \%$ \\
\hline Promedio & $12,12 \%$ & $4,14 \%$ \\
\hline
\end{tabular}

Fuente: INEI (2017) y ESCALE (2016).

* Gasto público en educación por alumno, superior universitaria

(soles corrientes).

. Producto Bruto Interno per cápita a precios constantes de 2007 (Soles).
Anexo 3. Convergencia

\begin{tabular}{|c|c|c|}
\hline $\begin{array}{l}\text { Regiones de Menor } \\
\text { Ingreso Per cápita } \\
2008\end{array}$ & $\begin{array}{l}\text { PBI per cápita (soles } \\
\text { 2007) }\end{array}$ & $\begin{array}{c}\text { Crecimiento } \\
\text { Económico } \\
\text { Promedio 2008-2015 }\end{array}$ \\
\hline Apurímac & 3825 & $4,42 \%$ \\
\hline Huánuco & 4266 & $5,20 \%$ \\
\hline Amazonas & 4725 & $5,35 \%$ \\
\hline San Martin & 4741 & $5,21 \%$ \\
\hline $\begin{array}{l}\text { Regiones de Mayor } \\
\text { Ingreso Per cápita } \\
2008\end{array}$ & $\begin{array}{l}\text { PBI per cápita (soles } \\
\text { 2007) }\end{array}$ & $\begin{array}{c}\text { Crecimiento } \\
\text { Económico } \\
\text { Promedio 2008-2015 }\end{array}$ \\
\hline Tacna & 16129 & $1,90 \%$ \\
\hline Madre de Dios & 16571 & $1,41 \%$ \\
\hline Pasco & 18814 & $-1,33 \%$ \\
\hline Moquegua & 51687 & $1,02 \%$ \\
\hline Nacional & 12111 & $4,14 \%$ \\
\hline Promedio regional & 11140 & $3,65 \%$ \\
\hline
\end{tabular}

